\title{
Viabilidad Económica de la Utilización de Biogás para la Conversión en Energía Eléctrica
}

\author{
Ricardo G. Cervi ${ }^{(1)}$, Maura S. T. Esperancini ${ }^{(2)}$ y Osmar de C. Bueno ${ }^{(3)}$ \\ (1) Faculdade de Tecnologia de Botucatu, Av. José Ítalo Bacchi, s/n - Jd. Aeroporto, \\ CEP: 18606-855, Botucatu, São Paulo-Brasil (e-mail: ricardogc@fca.unesp.br) \\ (2) Univ. Estadual Paulista, Fac. de Ciências Agronômicas, Depto. de Gestão e Tecnologia \\ Agroind., Rua José Barbosa de Barros 1780, CEP: 18603-970, Botucatu, São Paulo-Brasil \\ (e-mail: maura@fca.unesp.br) \\ (3) Univ. Estadual Paulista, Fac. de Ciências Agronômicas, Depto. de Gestão e Tecnologia \\ Agroind., Rua José Barbosa de Barros 1780, CEP: 18603-970, Botucatu, São Paulo-Brasil \\ (e-mail: osmar@fca.unesp.br)
}

Recibido Nov. 12, 2010; Aceptado Ene. 19, 2011; Versión Final recibida -Feb. 23, 2011

\begin{abstract}
Resumen
Se ha determinado la viabilidad económica para la conversión del biogás en electricidad, a partir del aprovechamiento de desechos de un criadero de cerdos. El biodigestor analizado es un modelo tubular continuo, con zanja para agua en albañilería y un contenedor cerrado, hermético e impermeable, donde son depositados diariamente los desechos de 2300 cerdos. La inversión inicial para implementación fue estimado en aproximadamente $\mathrm{R} \$ 51.537$ y los costos anuales del sistema fueron de $\mathrm{R} \$ 5.700$ con mantenimiento, $R \$ 4.400$ con depreciación y $R \$ 1.370$ con los intereses. Se encontró que un consumo medio de $28 \mathrm{~kW}$-hora ${ }^{-1}$ es el consumo mínimo que debe tener el sistema para ser económicamente factible.
\end{abstract}

Palabras clave: biogás, viabilidad económica, energía eléctrica, biodigestor, desechos de cerdos

\section{Economic Viability of using Biogas for Electrical Power Generation}

\begin{abstract}
The economic viability of the electrical power generation using biogas from swine wastes, was determined. The analyzed biodigester is a continuous tubular model with brick concrete duct and plastic covering with a gasometer, in which the waste of 2.300 fattening pigs are deposited daily. The initial investment estimate for the installation was $R \$ 51.537$, the system annual costs were $R \$$ 5.700 , for maintenance, $\mathrm{R} \$ 4.400$ for depreciation and $\mathrm{R} \$ 1.370$ for interests. It was found that an average consumption of $28 \mathrm{~kW}^{-}$hour $^{-1}$ is the minimum that the system must reach to be economically feasible.
\end{abstract}

Keywords: biogas, economic viability, electrical power, biodigester, swine wastes 


\section{INTRODUCCIÓN}

El creciente aumento en la actividad de criaderos de cerdos en Brasil y el incremento tecnológico en los sistemas de producción, resulta en un aumento en la generación de desechos, ocasionando problemas de orden sanitario, con daños a la salud, debido al alto contenido de substancias patogénicas presentes en esos residuos. Cuando es bien conducido, el manejo permite el aprovechamiento integral de los desechos, dentro de las condiciones establecidas en cada propiedad (Gaspar, 2003). En el medio rural, los sistemas biointegrados, específicamente con aprovechamiento de biomasa para fines energéticos, pueden convertirse en un medio facilitador para atingir la sustentabilidad de la producción en función de la disponibilidad de biomasa existente en las propiedades agrícolas, por presentar bajo costo de oportunidad de los residuos de la producción, grande potencial de generación de energía, disminución del potencial de polución de los residuos, reducción de la presión sobre os recursos naturales e economía de recursos energéticos (Angonese et al, 2006).

De ese modo, el tratamiento de esos efluentes puede realizarse a través de la biodigestión anaeróbica que permite valorizar un producto energético, el biogás, y obtener bioabono, cuja disponibilidad contribuye para una rápida amortización de los costos de la tecnología instalada (Pecora, 2006). Para crear sistemas de biodigestores económicamente más eficientes, es necesario analizar la ejecución del proyecto y el manejo de la tecnología adoptada, que pueden permitir la construcción de instalaciones más económicas y recuperación de los invertimientos de forma más rápida. De ese modo son evaluados correctamente los beneficios energéticos del biogás, del reciclaje del efluente como abono y del saneamiento (Miranda, 1991). Para que estos sistemas sean adoptados es fundamental que haya lucro económico para los empresarios. Diversos estudios han sido realizados para identificar la viabilidad económica de sistemas de biodigestión anaeróbica de desechos y de residuos de la producción. Nogueira y Zürn (2005) al estudiar el desarrollo de un nuevo modelo para dimensionamiento de sistemas integrados de recursos energéticos renovables, aplicados en ambientes rurales, con la utilización de herramientas de simulación y optimización, concluyeron que el dimensionamiento de los sistemas energéticos y sus costos son fundamentales para la tomada de decisiones en proyectos de electrificación rural, considerando criterios técnicos y económicos.

Lozano Y Ramos (2007) señalan que a pesar de las iniciativas acordadas por los gobiernos y de los esfuerzos de las empresas, las emisiones de $\mathrm{CO}_{2}$ aumentaron un $20 \%$ durante la última década. La posibilidad de crear fuentes que abastezcan, que sean descentralizadas y en pequeña escala es fundamental para el desarrollo sustentable. Centrales que utilicen fuentes renovables que no demanden alta tecnología para su instalación o mano de obra especializada para su ejecución, son soluciones direccionadas principalmente para productores rurales que podrían disminuir su dependencia de energía eléctrica de las empresas concesionarias (Coldebella, 2006). La quema de biogás sustituyendo las emisiones de metano del dióxido de carbono esválida, ya que el metano tiene un potencial de calentamiento global 21 veces mayor que el dióxido de carbono (Ranzi y Andrade, 2004). Siendo así, este trabajo tuvo como objetivo identificar los costos y beneficios de una planta instalada de aprovechamiento de desechos de cerdos para producción de biogás y posterior conversión en energía eléctrica en unidad productiva biointegrada, en la Región de Botucatu-SP y analizar la viabilidad económica del sistema, a partir de la utilización actual de la energía eléctrica generada, bien como identificar el consumo mínimo necesario de energía para viabilizar económicamente el sistema.

\section{MATERIALES Y MÉTODOS}

Este estudio fue realizado en una Agroindustria, localizada en el municipio de São Manuel, Estado de São Paulo, situada a una latitud de $22^{\circ} 43^{\prime} 50^{\prime \prime}$ Sur y longitud de $48^{\circ} 34^{\prime} 14^{\prime \prime}$ Oeste. El clima de la región es del tipo Cfa (Método de Köeppen), clima temperado caluroso, mesotérmico y Húmedo (Cunha y Martins, 2009). La principal actividad de la propiedad es la crianza de gallinas ponedoras. Además de ésta, son realizadas otras actividades agrícolas, tales como el cultivo de café, crianza de cerdos con 2.300 animales en fase de crecimiento y terminación, y crianza de ovinos y bovinos, en menor escala. El biodigestor analizado en este estudio tiene el 
formato tubular y operación continua, con zanja para agua en albañilería y un contenedor cerrado, hermético e impermeable (llamado reactor), representado en la Figura 1.

El biodigestor posee el formato de tronco de pirámide inferior. Los datos de la Tabla 1 muestran los parámetros dimensionales de construcción del biodigestor, indicando un volumen útil de 496 $\mathrm{m}^{3}$.

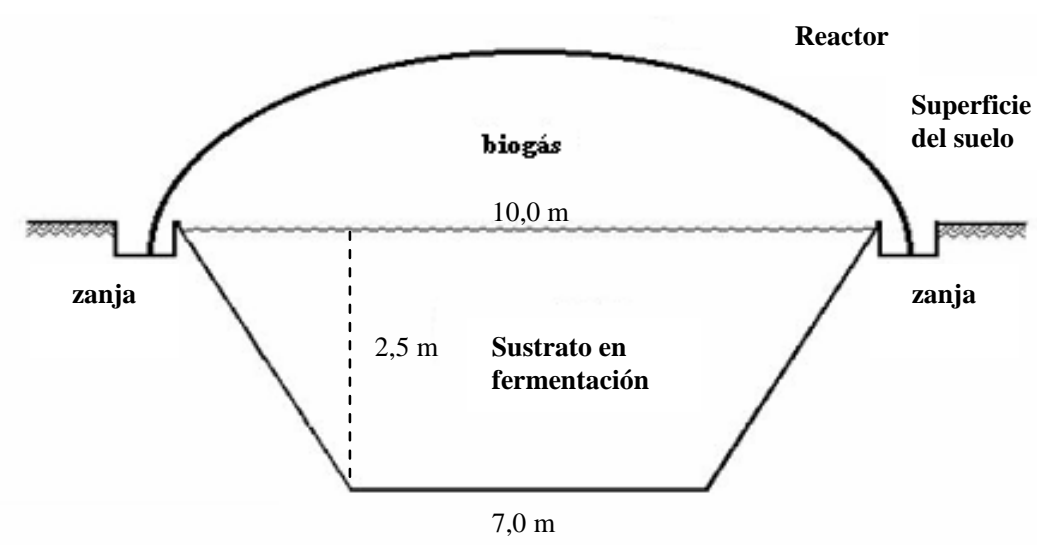

Fig. 1: Sección transversal del biodigestor.

Tabla 1: Dimensiones del biodigestor, modelo tubular, con contenedor cerrado, hermético e impermeable (llamado reactor)

\begin{tabular}{|l|c|c|c|}
\hline Dimensiones & Unidad & Volumen de Excavación & Volumen Útil \\
\hline Altura & metros & 2,8 & 2,5 \\
\hline Anchura Inferior & metros & 7 & 7 \\
\hline Largura inferior & metros & 22 & 22 \\
\hline Anchura Superior & metros & 10 & 10 \\
\hline Largura Superior & metros & 25 & 25 \\
\hline Área Inferior & $\mathrm{m}^{2}$ & 154 & 154 \\
\hline Área Superior & $\mathrm{m}^{2}$ & 258 & 246 \\
\hline Volumen & $\mathrm{m}^{3}$ & 571 & 496 \\
\hline
\end{tabular}

Los desechos generados por los cerdos son conducidos para una caja de captación donde ocurre la homogeneización del flujo, y luego son enviados directamente para el biodigestor. Para 2.300 cerdos en fase de terminación la producción diaria es de $1.837,7 \mathrm{~m}^{3}$ de biogás, producción que fue estimada con base en Oliveira (1993) que determinó que para cerdos en fase de terminación con peso entre 25 y $100 \mathrm{~kg}$ son producidos $7 \mathrm{~L}$. día ${ }^{-1}$ de desechos líquidos, en promedio, y los datos de Santos (2000) que indican que cada cerdo en fase de terminación produce $0,799 \mathrm{~m}^{3}$ diarios de biogás. Siendo así, podemos estimar que 2.300 cerdos en fase de terminación producen aproximadamente 16,10 $\mathrm{m}^{3}$ de desechos por día.

El consumo específico de biogás por grupo generador fue estimado en $22 \mathrm{~m}^{3} \cdot \mathrm{h}^{-1}$, relación fornecida por el fabricante del motor-generador, dada por el volumen de biogás consumido por el grupo generador y la energía eléctrica generada. El generador de electricidad es trifásico, 220/380 VCA, 3.600 RPM, $60 \mathrm{~Hz}$, con capacidad nominal de generación de 50kVA. Conforme la norma 
NBR 5410 de la Asociación Brasileña de Normas Técnicas- ABNT (2004), para cálculos de generación de energía eléctrica en baja tensión, para un factor de potencia medio de carga de 0,8 $(\operatorname{COS} \varnothing)$, la generación máxima de energía es de $40 \mathrm{kw}$. El equipo posee control de rotación electrónico del tipo isócrono, con sensor electromagnético, y dispone de protección contra sub y sobre velocidad.

El generador fue acoplado a un motor de 2000 CC, de 4 cilindros, adaptado para utilizar biogás. Fue hecho un muestreo de los datos referentes a la potencia activa (KW) consumida, con la utilización del analizador de rede Circutor AR5- ${ }^{\circledR}$ que posee conjunto de cabos flexibles de 6.000 Amperes, con registros de intervalos de 10 minutos en cada medición. Los registros fueron monitoreados por 7 días, intervalo considerado suficiente, una vez que se verificó que no existen variaciones significativas entre semanas. La evaluación económica del sistema es compuesta de 3 etapas, como se describe en lo que sigue.

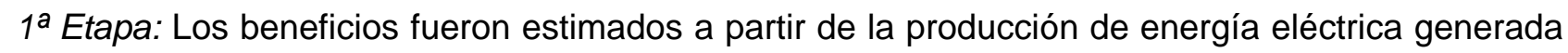
en el sistema y consumida en la propiedad, o sea, es la renta que se deja de transferir para la concesionaria de energía eléctrica por no adquirir energía externa para la propiedad. La agroindustria, de acuerdo a la clasificación de la concesionaria de energía, está clasificada con tarifa "horo-sazonal verde", que se caracteriza por la aplicación de tarifas diferenciadas en horarios de punta (de 18:00 a 21:00h) o fuera de horas de mayor demanda (las demás horas del día) para el consumo de energía. (Companhia Paulista de Força e Luz, 2008).

De esta manera, el beneficio es dado por la cantidad de energía eléctrica producida por el sistema y consumida en la propiedad de acuerdo al tiempo de operación de la planta y de la tarifa de energía eléctrica que paga el propietario (Ecuación 1):

$$
B G E E=(E E C X T) T E E
$$

Donde:

BGEE - Beneficio con la generación de energía eléctrica $(R \$ . a n ̃ o-1)$.

EEC - Energía eléctrica consumida $\left(\mathrm{kW} \cdot\right.$ hora $\left.^{-1}\right)$.

$\mathrm{T}$ - Tiempo de operación (horas.año-1 $)$.

TEE - Tarifa de energía eléctrica (R\$.kW).

2a Etapa: Los costos fueron estimados considerando los costos fijos oriundos del invertimiento inicial y de los costos variables de mantenimiento del sistema. La inversión inicial es clasificada como el gasto necesario para la implantación de las instalaciones y adquisiciones de los materiales y equipamientos. Estos datos fueron colectados en la propiedad, y en algunos casos, fueron realizadas cotizaciones de mercado. La inmovilización del capital para el invertimiento inicial genera costos a lo largo de la vida útil del sistema, que pueden ser colocados a disposición anualmente. Estos costos son depreciación e intereses sobre el capital invertido.

El costo de depreciación fue estimado por el método de la depreciación linear conforme Nogueira (2001), dado por la Ecuación 2:

$D=\frac{V i-V f}{V u}$

Donde:

$\mathrm{D}$ - Depreciación anual $\left(\mathrm{R} \$ . \mathrm{año}^{-1}\right)$.

$\mathrm{Vi}$ - Valor inicial de los bienes de capital $(R \$)$.

$\mathrm{Vf}$ - Valor final de los bienes de capital $(\mathrm{R} \$)$.

Vu - Vida útil (años).

El interés sobre el capital invertido fue determinado considerando el capital medio durante la vida útil de los bienes con una tasa de interés anual de 5,64\% al año, conforme el rendimiento anual de 
aplicación de bajo riesgo, para evitar estimativas supervaloradas de este ítem de costo, que equivale a una tasa de interés compuestos de $0,5 \%$ al mes, aplicada sobre los valores actualizados por la Tasa Referencial (TR). Según el método descripto por Nogueira (2001) el costo de oportunidad del capital puede ser estimado usando la ecuación 3.

$$
V k=\frac{V i+V f}{2} \times r
$$

Donde:

$\mathrm{Vk}$ - Costo de oportunidad del capital $\left(\mathrm{R} \$ . \mathrm{año}^{-1}\right)$.

$\mathrm{Vi}$ - Valor inicial de los bienes de capital $(R \$)$.

$\mathrm{Vf}$ - Valor final de los bienes de capital $(\mathrm{R} \$)$.

$r$ - Tasa de intereses anuales $\left(\% \cdot a n ̃ 0^{-1}\right)$.

Los costos de mantenimiento son costos variables oriundos del mantenimiento del grupo generador y los gastos referentes a la mano de obra para operar el sistema. Para estimar el costo de mantenimiento do grupo generador fue considerado el intervalo de mantenimiento de los componentes como cambio de aceite, lubrificación etc., conforme el Manual del Equipamiento fornecido por el fabricante, de acuerdo al tiempo de operación del grupo generador. Estos costos fueron basados en los valores cobrados por la asistencia técnica por estos servicios en la región incluyendo materiales utilizados y los impuestos. De esta manera, el gasto anual con el mantenimiento del grupo generador fue definido por la Ecuación 4.

$G M G G=\left(\frac{T}{I M}\right) A T$

Donde:

GMGG - Gastos con el mantenimiento del grupo generador (R\$.año-1).

$\mathrm{T}$ - Tiempo de operación (horas.año ${ }^{-1}$ ).

IM - Intervalo de mantenimiento de los componentes (horas.unidad ${ }^{-1}$ ).

AT - Asistencia técnica $\left(R \$\right.$. unidad $\left.^{-1}\right)$.

La operación del grupo generador es diaria y exige la presencia de una persona responsable por la ignición de motor, limpieza y para mantener en orden las instalaciones. El costo de la mano de obra para mantener el biodigestor en operación es muy bajo debido a la simplicidad del sistema. Es necesaria apenas una limpieza para remover el lodo precipitado en el biodigestor y la costra que se forma en la superficie. Así, para estimar el costo de la mano de obra necesaria para el mantenimiento fue considerado el tiempo de operación exigido en función del salario pagado, conforme la Ecuación 5:

$G M O=T O x G S$

Donde:

GMO - Gastos con mano de obra para operación del sistema (R\$.año ${ }^{-1}$ ).

TO - Tiempo de operación exigido (horas.año ${ }^{-1}$ ).

GS - Gastos con salario $\left(\mathrm{R} \$ \cdot\right.$ hora $\left.^{-1}\right)$.

Los beneficios del sistema de conversión de biogás y los costos son dados anualmente y pueden ser comparados entre si.

$3^{a}$ Etapa - Establecidos los beneficios económicos y los costos fijos y variables anuales, es posible establecer el punto mínimo de consumo de energía, a partir del cual el empresario debe 
actuar para evitar la operación de la actividad en escala menor a la económicamente eficiente. Este punto es conocido como punto de equilibrio y puede ser comparado con la utilización actual e identificar si los niveles de consumo de energía están arriba o abajo del punto de equilibrio. Bruni (2006) señala que en el punto de equilibrio el lucro es nulo. La receta total es igual al precio multiplicado por la cantidad y los costos totales son iguales al costo fijo más el costo variable unitario multiplicado por la cantidad. Así, la cantidad a ser producida y comercializada para un lucro nulo, puede ser representada por la ecuación 6: El Costo Variable Unitario (CVU) es dado por la ecuación 7:

$P E=\frac{\frac{C F}{P-C V U}}{T}$

Donde:

PE - Punto de Equilibrio en cantidad de energía consumida (kW.hora ${ }^{-1}$ ).

$\mathrm{CF}$ - Costo con depreciación e intereses $\left(\mathrm{R} \$ . \mathrm{año} \mathrm{o}^{-1}\right)$.

$\mathrm{P}$ - Tarifa de energía eléctrica $\left(\mathrm{R} \$ . \mathrm{kW}^{-1}\right)$.

$\mathrm{T}$ - Tiempo de operación (horas.año ${ }^{-1}$ ).

CVU - Costo Variable Unitario.

$C V U=\frac{C V O M}{C A E}$

Donde:

$\mathrm{CVU}$ - Costo variable unitario $\left(\mathrm{R} \$ . \mathrm{kW}^{-1}\right)$.

CVOM - Costo variable unitario con mantenimiento y operación (R\$.año ${ }^{-1}$ )

$\mathrm{CAE}$ - Consumo anual de energía eléctrica $\left(\mathrm{kW} . \mathrm{año}^{-1}\right)$.

La representación gráfica de los puntos de equilibrio en cantidad, puede ser vista en la figura 2.

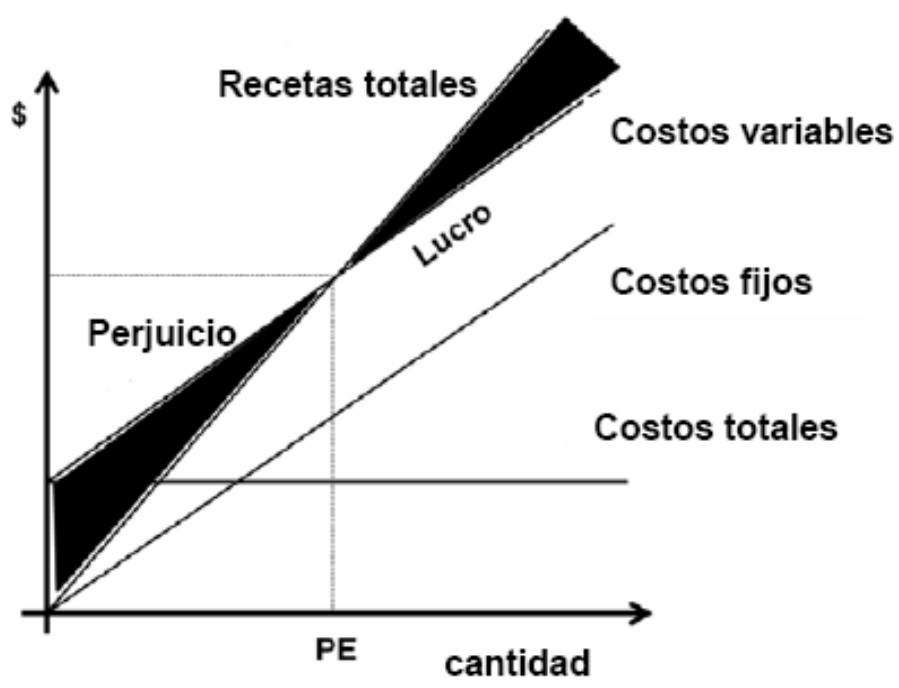

Fig. 2: Representación gráfica del punto de equilibrio.

\section{RESULTADOS}

La producción de biogás depende directamente de las condiciones de mantenimiento y manejo del biodigestor y del residuo. La producción de biogás fue estimada en $670.760,5 \mathrm{~m}^{3}$.año-1 . El biogás producido fue utilizado directamente en el sistema de conversión de energía eléctrica, que opera 
durante 10,5 horas.día $^{-1}$, en media, excepto los domingos y feriados. De esta manera, fue estimado un período de 26 días por mes y 312 días de operación por año, que resultó en 3.276 horas de operación por año. El consumo de biogás fue de $72.072 \mathrm{~m}^{3}$. año ${ }^{-1}$. Este consumo representó aproximadamente $10,74 \%$ del volumen de biogás producido por la planta. El conjunto motor-generador opera, en media, desde las 7:00 hasta las 17:30h. De esa manera fue considerada la tarifa cobrada en el horario fuera de punta que es de $\mathrm{R} \$ 0,165 . \mathrm{kW}^{-1}$, conforme datos de la propiedad. El beneficio con la generación de energía eléctrica fue estimado considerando el consumo medio de energía eléctrica en función del número de horas de operación del grupo generador. Conforme los datos obtenidos (Figura 3) a través de las mediciones realizadas por el analizador de red, el consumo medio diario fue de $17,1 \mathrm{~kW} . \mathrm{h}^{-1}$.

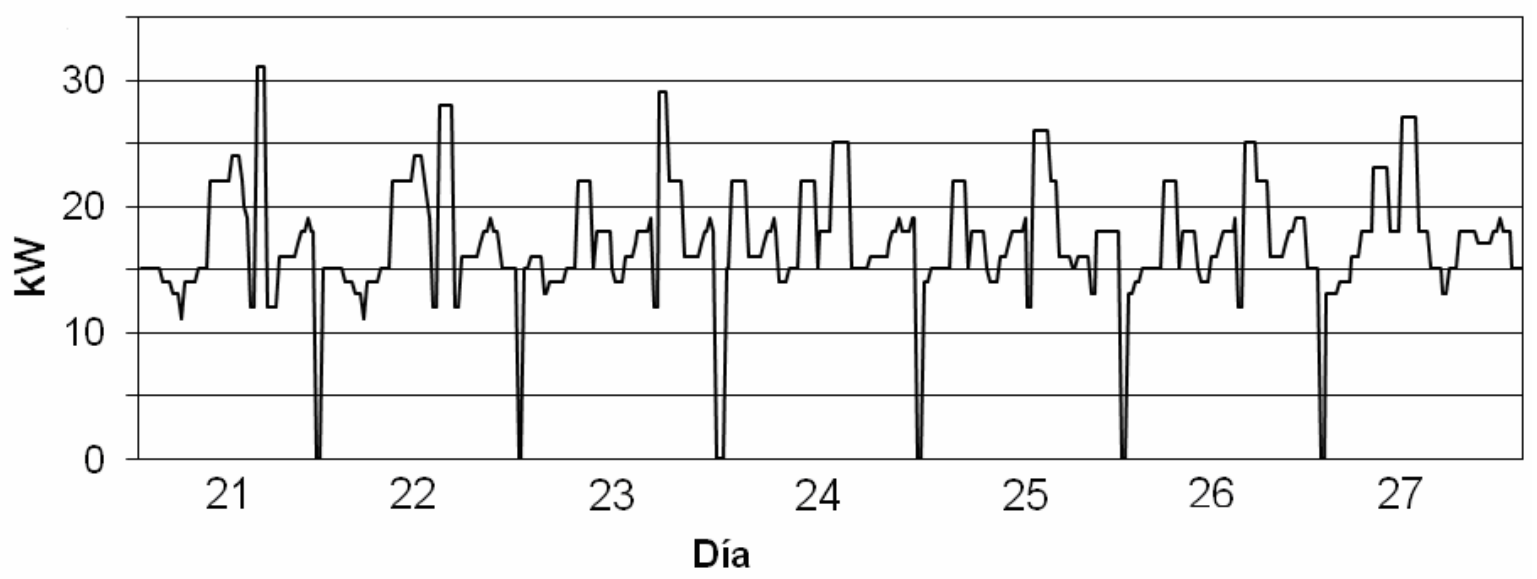

Fig. 3: Mediciones del consumo de energía eléctrica.

El generador presentó el parámetro de tensión trifásica de 220V. La corriente máxima a ser solicitada por la carga instalada puede ser de 131,82 A. No obstante, a pesar de que el grupo generador disponibilice $40 \mathrm{~kW}$ de consumo máximo, las mediciones indican que el consumo promedio es de $17,1 \mathrm{KW}$, o sea, 56,35 A. Por lo tanto, utiliza en media apenas $43 \%$ de la energía generada disponible por el generador. La tabla 2 muestra el invertimiento inicial con los respectivos ítem de costo y valores de adquisición, construcción y servicios referentes a la implantación del sistema. Los costos de depreciación, intereses sobre el capital invertido, y costos de mantenimiento y operación son representados en las Tablas 3 a 5, respectivamente. En las Tablas 3 y 4 el valor final de los bienes fueron considerados de valor cero al final de su vida útil.

Tabla 2: Invertimiento inicial para un sistema generador de energía eléctrica producida a partir de biogás.

\begin{tabular}{|l|r|}
\hline Ítems & \multicolumn{1}{c|}{$(\mathrm{R} \$)$} \\
\hline Adquisición del grupo generador & $28.594,00$ \\
\hline Construcción del biodigestor & $15.276,77$ \\
\hline $\begin{array}{l}\text { Construcción del abrigo del grupo } \\
\text { generador }\end{array}$ & 785,95 \\
\hline Instalaciones eléctricas & $4.110,45$ \\
\hline Mano de obra para implantación & $2.770,00$ \\
\hline Total & $51.537,17$ \\
\hline
\end{tabular}


Tabla 3: Costos anuales de depreciación de los bienes utilizados en la implantación de un sistema generador de energía eléctrica a partir de biogás.

\begin{tabular}{|l|r|c|r|}
\hline \multicolumn{1}{|c|}{ Costos de depreciación* } & Valor inicial & Vida útil & Depreciación \\
\cline { 2 - 4 } & $(\mathrm{R} \$)$ & $(\mathrm{años})$ & \multicolumn{1}{c|}{$\left(\mathrm{R} \$ . \mathrm{año}^{-1}\right)$} \\
\hline $\begin{array}{l}\text { Biodigestor Albañilería y } \\
\text { otros materiales }\end{array}$ & $8.340,35$ & 20 & 417,02 \\
\hline $\begin{array}{l}\text { Abrigo del conjunto motor- } \\
\text { generador }\end{array}$ & 785,95 & 20 & 39,30 \\
\hline Reactor del biodigestor & $6.636,42$ & 10 & 663,64 \\
\hline Instalaciones eléctricas & $4.110,45$ & 10 & 411,05 \\
\hline Grupo generador & $28.594,00$ & 10 & $2.859,40$ \\
\hline Total & & & $4.390,40$ \\
\hline
\end{tabular}

Tabla 4: Intereses sobre el capital invertido en los bienes de capital utilizados en la implantación de un sistema generador de energía eléctrica a partir de biogás.

\begin{tabular}{|l|r|c|r|}
\hline \multirow{2}{*}{ Capital invertido } & Valor inicial & Tasa de intereses & intereses \\
\cline { 2 - 4 } & $(\mathrm{R} \$)$ & $\left(\%\right.$. año $\left.{ }^{-1}\right)$ & $\left(\mathrm{R} \$ . \mathrm{año}^{-1}\right)$ \\
\hline Biodigestor Albañilería y otros materiales & $8.340,35$ & 5,64 & 235,20 \\
\hline Abrigo del conjunto motor-generador & 785,95 & 5,64 & 22,16 \\
\hline Reactor del biodigestor & $6.636,42$ & 5,64 & 187,15 \\
\hline Instalaciones eléctricas & $4.110,45$ & 5,64 & 115,91 \\
\hline Grupo generador & $28.594,00$ & 5,64 & 806,35 \\
\hline Total & & & $1.366,77$ \\
\hline
\end{tabular}

Tabla 5: Mantenimiento preventivo del grupo generador de energía eléctrica a partir de biogás.

\begin{tabular}{|c|c|c|c|}
\hline Componente & Intervalo (horas) & $\begin{array}{c}\text { Costo de operación y } \\
\text { mantenimiento }(R \$)\end{array}$ & $\begin{array}{c}\text { Costo anual de } \\
\text { operación y } \\
\text { mantenimiento (R\$) }\end{array}$ \\
\hline $\begin{array}{l}\text { Aceite } \\
\text { lubricante }\end{array}$ & $\begin{array}{l}\text { Cambio de aceite a cada } \\
100 \text { horas }\end{array}$ & 80,00 & $2.620,80$ \\
\hline Filtro de aceite & $\begin{array}{l}\text { Cambio de filtro de aceite a } \\
\text { cada } 400 \text { horas }\end{array}$ & 52,00 & 425,88 \\
\hline \multirow{2}{*}{$\begin{array}{l}\text { Sistema de } \\
\text { combustible }\end{array}$} & $\begin{array}{l}\text { Limpieza de los filtros a } \\
\text { cada } 200 \text { horas }\end{array}$ & 15,00 & 245,70 \\
\hline & $\begin{array}{l}\text { Limpieza de la válvula de } \\
\text { gas a cada } 2.000 \text { horas }\end{array}$ & 15,00 & 24,57 \\
\hline
\end{tabular}


Tabla 5: (continuación)

\begin{tabular}{|l|l|c|c|}
\hline \multirow{2}{*}{ Filtro de aire } & $\begin{array}{l}\text { Limpieza a cada 1.000 } \\
\text { horas }\end{array}$ & 15,00 & 49,14 \\
\cline { 2 - 4 } & $\begin{array}{l}\text { Cambio do filtro de aire a } \\
\text { cada 2.000 horas }\end{array}$ & 86,00 & 140,87 \\
\hline \multirow{2}{*}{$\begin{array}{l}\text { Sistema de } \\
\text { refrigeración }\end{array}$} & $\begin{array}{l}\text { Cambio del Fluido } \\
\text { refrigerante, de la correa } \\
\text { dentada y del estirador de } \\
\text { la correa a cada 1.000 hrs. }\end{array}$ & 220,00 & 720,72 \\
\hline \multirow{2}{*}{ Alternador } & $\begin{array}{l}\text { Cambio de la correa y del } \\
\text { juego de bujías a cada } \\
1.000 \text { horas }\end{array}$ & 200,00 & 655,20 \\
\cline { 2 - 4 } & $\begin{array}{l}\text { Cambio de los rodamientos } \\
\text { a cada 2.000 horas }\end{array}$ & 100,00 & 163,80 \\
\hline $\begin{array}{l}\text { Rodamiento del } \\
\text { generador }\end{array}$ & Lubricar a cada 1.000 horas & 20,00 & 65,52 \\
\hline Total & & & $5.112,20$ \\
\hline
\end{tabular}

Para la estimativa de la mano de obra necesaria para el mantenimiento fue considerado el tiempo de operación exigido estimado en 200 horas.año $^{-1}$ y el salario pagado de $R \$ 2,98$. hora ${ }^{-1}$, que resultó en $\mathrm{R} \$ 596,00$.año ${ }^{-1}$. De esta forma, los costos totales con mantenimiento y operación del biodigestor y del grupo generador fueron de $\mathrm{R} \$ 5.708,20 . \mathrm{año}^{-1}$. Ese costo representa aproximadamente $11,08 \%$ del invertimiento inicial para la implantación del sistema.

\section{DISCUSIÓN}

Para el consumo medio anual de energía producida por el sistema generador de 56.019,60 kW.año ${ }^{-1}$, el beneficio anual fue de $R \$ 9.243,23 . a n ̃ o^{-1}$, frente a un costo anual total de $R \$$ 10.835,37.año $0^{-1}$, o sea, los costos son aproximadamente $14,69 \%$ superiores a los beneficios, indicando que el empresario, en este nivel de consumo, tiene un perjuicio anual de $\mathrm{R} \$$ 1.592,14.año-1.

Fue identificado el punto de equilibrio en cantidad de energía consumida como siendo de 27,85 kW.hora ${ }^{-1}$, o sea, apenas para cubrir todos los costos envueltos, considerando el precio de la tarifa de energía de $R \$ 0,165 . \mathrm{kW}^{-1}$, costo variable medio de $R \$ 0,102 \mathrm{~kW}^{-1}$, y costo fijo de $R \$$ $5.757,17$.año ${ }^{-1}$, para un tiempo estimado de utilización del generador de 3.276 horas.año ${ }^{-1}$ Eso muestra que para el consumo diario actual de $17,1 \mathrm{~kW} \cdot$ hora $^{-1}$ el sistema presenta perjuicio y debe presentar retorno financiero positivo si el consumo medio fuera superior a 27,85 kW.hora ${ }^{-1}$, considerando la misma operación media de 10,5 horas. día ${ }^{-1}$

Souza et al. (2004) estudiando una propiedad rural típica compuesta de gallinero, pocilga, fábrica de ración y residencia estimaron una carga utilizada estimada en $39 \mathrm{~kW}$. De esa manera, constataron que serían necesarias 258 matrices de cerdos con capacidad de producir, cada una, $0,775 \mathrm{~m}^{3}$ de biogás por día, para poder instalar un grupo generador de energía de $40 \mathrm{~kW}$. Para el análisis económico consideraron una tasa de descuento de $8 \%$ al año, y costos de operaciónmantenimiento de $4 \%$ del invertimiento inicial para la implantación del sistema.

Zago (2003) evaluó el potencial de producción de energía eléctrica a través del biogás, en la región del Medio Oeste de Santa Catarina, y constató que para un criadero de credos con producción media de $50 \mathrm{~m}^{3}$ de biogás.día ${ }^{-1}$ posee capacidad de generar aproximadamente 2.160 kWh.mes. ${ }^{-1}$ de energía eléctrica. También observó que con la utilización de un sistema que sea 
capaz de generar $25 \mathrm{KVA} \cdot \mathrm{h}^{-1}$ de potencia eléctrica, las propiedades pueden convertirse en autosuficientes en energía eléctrica. El consumo de biogás observado por el autor varía de 16 a $25 \mathrm{~m}^{3}$.hora ${ }^{-1}$ para el grupo generador estacionario para la generación de energía eléctrica y que eso depende de la potencia eléctrica generada. El estudio destacó que el emprendimiento pasa a ser viable económicamente cuando la propiedad posee capacidad de producción de $200 \mathrm{~m}^{3}$.día ${ }^{-1}$ de biogás que puede generar aproximadamente $300 \mathrm{kVAh}$ día $^{-1}$.

\section{CONCLUSIÓN}

Sistemas de biodigestión anaeróbica pueden ser considerados instrumentos importantes de racionalización de la producción y del uso de energías renovables en la agricultura, principalmente en escalas menores de producción. La adopción de estos sistemas por los productores rurales depende de su viabilidad técnica y económica. Se verificó en este estudio la importancia de establecer parámetros económicos para la decisión en este tipo de invertimiento. Como son generados excedentes de energía eléctrica, y biogás que no son aprovechados por el sistema de conversión de biogás en energía eléctrica, y ni son vendidos, la viabilidad económica del sistema queda comprometida, indicando que el sistema fue superdimensionado en su estructuración de costos ante los beneficios proporcionados y que el dimensionamiento técnico debe ser acompañado de evaluaciones económicas para que el sistema sea sustentable del punto de vista económico. En este caso, se puede recomendar la utilización de un grupo generador de energía eléctrica superior a su punto de equilibrio para que el sistema genere beneficios financieros.

\section{REFERENCIAS}

Angonese, A.; Campos, A. T.; Zacarkim, C. E. Eficiência energética de sistema de produção de suínos con tratamento dos resíduos em biodigestor. Revista Brasileira Engenharia Agrícola e Ambiental: 10(3), 745-750, Campina Grande-PB, Brasil (2006).

Associação Brasileira de Normas Técnicas (Brasil). ABNT NBR5410: Instalações elétricas de baixa tensão, São Paulo, http://www.pdf-search-engine.com/download-normas-nbr-pdf.html. Acesso: 27 de Abril (2009).

Bruni, A. L. Administração de custos, preços e lucros. Série desvendando as finanças v.5. 68-88, Atlas, São Paulo, Brasil (2006).

Coldebella, A. Viabilidade do uso do biogás da bovinocultura e suinocultura para geração de energía elétrica e irrigação em propriedades rurais. 73p. Dissertação de Mestrado. Mestrado em Engenharia Agrícola/Engenharia de Sistemas Agroindustriais, Universidade Estadual do Oeste do Paraná (UNIOESTE), Cascavel, Brasil (2006).

Companhia Paulista de Força e Luz. Taxas e tarifas. Campinas, http://agencia.cpfl.com.br/portalservicos/paulista/taxas_tarifas.asp. Acesso: 31 de Outubro (2008).

Cunha, A.R.; Martins, D. Classificação climática para os municípios de Botucatu e São Manuel, SP. Revista Irriga: (14)1,1-11, Botucatu-SP, Brasil (2009).

Gaspar, R.M.B.L. Utilização de biodigestores em pequenas e médias propriedades rurais con ênfase na agregação de valor: un estudo de caso na região de Toledo - PR. 106p. Dissertação de Mestrado. Mestrado em Planejamento e Estratégia Organizacional, Universidade Federal de Santa Catarina (UFCS), Florianópolis-SC, Brasil (2003).

Lozano, Miguel A; Ramos, José. Análisis Energético y Económico de Sistemas Simples de Cogeneración. Inf. tecnol., La Serena, v. 18, n. 5, 2007. Disponible en

$<$ http://www.scielo.cl/scielo.php?script=sci_arttext\&pid=S0718-

07642007000500010\&lng=es\&nrm=iso>. accedido en 27 enero 2011. 
Miranda, H. A. Influência da recirculação de efluentes e do tempo de retenção no desempenho de biodigestores operados con estrume de suínos. 137p. Dissertação de Mestrado. Mestrado em Agronomia/Energía na Agricultura, Faculdade de Ciências Agronômicas, Universidade Estadual Paulista (UNESP), Botucatu-SP, Brasil (1991).

Nogueira, C. E. C., Zürn, H. H.. Modelo de dimensionamento otimizado para sistemas energéticos renováveis em ambientes rurais. Revista Engenharia Agrícola, (25)2, 341-348, Jaboticabal-SP, Brasil (2005).

Nogueira, E. Análise de investimentos. In: Gestão agroindustrial por BATALHA, M. O: Atlas, 2 ed., 556-584, São Paulo-SP, Brasil (2001).

Oliveira, P. A. V. Manual de manejo e utilización dos dejetos de suínos.188p. Empresa Brasileira de Pesquisa Agropecuária (EMBRAPA), Centro Nacional Suínos e Aves, Concórdia-SC, Brasil (1993).

Pecora, V. Implantação de uma unidade demonstrativa de geração de energía elétrica a partir do biogás de tratamento do esgoto residencial da USP: estudo de caso. 152p. Dissertação de Mestrado. Mestrado em Energía, Universidade de São Paulo (USP), São Paulo-SP, Brasil (2006).

Ranzi, T. J. D.; Andrade, M. A. N. Estudo de viabilidade de transformação de esterqueiras e bioesterqueiras para dejetos de suínos em biodigestores rurais visando o aproveitamento do biofertilizante e do biogás. In: Encontro de energia no meio rural e geração distribuída, 5., 2004, Campinas. Anais... Campinas: Universidade Estadual de Campinas, 2004. 1 CD-ROM.

Santos, P. Guia técnico de biogás. 117 p. Centro para a Conservação de Energía, Espinho, Portugal (2000).

Souza, S. N. M., Pereira, W. C., Nogueira, C. E. C., Pavan, A. A., Sordi, A. Custo da eletricidade gerada em conjunto motor gerador utilizando biogás da suinocultura. Acta Scientiarum. (26)1, 127133, Maringá-PR, Brasil (2004).

Zago, S. Potencialidade de produção de energía através do biogás integrada à melhoria ambiental em propriedades rurais con criação intensiva de animais, na região do meio oeste catarinense. 103p. Dissertação de Mestrado, Mestrado em Engenharia Ambiental, Universidade Regional de Blumenau (URB), Blumenau-SC, Brasil (2003). 
